\title{
Two-Dimensional Wavelets with Complementary Filter Banks
}

\author{
M.G. ALMEIDA ${ }^{1}$, CEFET-MG / DPPG, Av. Amazonas 7675, 30510-000 Belo
} Horizonte, MG, Brazil.

\begin{abstract}
In this paper, the Two-Dimensional (2D) Complementary Filter (CF) Banks, a signal processing technique which can be used to get scaling and wavelets functions, are presented. The $2 \mathrm{D}$ multirate signal processing theory and complementary filters properties are the base of CF Banks, permitting us to consider different types of sampling and filters. Two-dimensional nonseparable quincunx, rectangular and circular complementary filters are designed for an alias free decimation and interpolation. When CF bank is implemented with quincunx sampling and filters, perfect reconstruction is achieved and although it is not reached in others cases, the analysis and synthesis are performed without aliasing. The CF banks are related with wavelet theory and procedures to get $2 \mathrm{D}$ scaling and bandpass wavelets functions from the L-level filter bank tree structure iteratively are shown. We illustrate scaling and wavelets functions convergence as the level of decomposition increases.
\end{abstract}

\section{Introduction}

Signal processing techniques have many applications in data compression, bandwidth reduction, edge detection, analysis/synthesis of signals. In this paper, the $\mathrm{CF}$ banks, a signal processing technique developed originally for the processing of images [1], is used to get $2 \mathrm{D}$ scaling and wavelets functions. The wavelet theory applied with image processing introduced by Mallats [2, 3] used one-dimensional wavelets and quadrature mirror filters. We had interest in its extension to nonseparable bank of filters considering the strong link that exists between wavelet theory and multirate processing theory. The link between wavelet theory, bank of filter and multirate processing $[4,5]$ was shown before. The theory of bank of filters using quincunx mirror filters and its connection with wavelet theory was introduced in [4].

In two-dimensions, complementary and mirror filters differ from each other because lowpass and highpass complementary filters cover entirely the 2D spectrum while with exception of quincunx mirror filters, lowpass and highpass mirror filters do not cover completely the 2D spectrum [1]. The CF Banks allow scaling and

${ }^{1}$ graca@dppg.cefetmg.br 
bandpass wavelet functions to be obtained straightforward. Through Noble's identities [5], a general expression for the equivalent transfer function in L-levels of CF banks decomposition was found [1]. A similar expression was shown in Vetterli at al. [4]. We denote these bandpass wavelets since they are related with the ithlevel bandpass filters of the L-level complementary filter banks tree. Similarly, the $2 \mathrm{D}$ scaling function is related to the ith-equivalent lowpass filter in the L-level CF Banks tree.

The rest of the paper is organized as follows. In section 2, the basic of $2 \mathrm{D}$ multirate signal processing theory is introduced. In section 3, the complementary filter design, CF banks and wavelet representation are presented. In section 4, procedures to get $2 \mathrm{D}$ scaling and wavelets functions iteratively and an illustration of their convergence as the level of decomposition increases are shown. Finally, in section 5, we make the conclusions.

\section{Basic Two-Dimensional Multirate Signal Pro- cessing}

In this section, we introduce the basics of $2 \mathrm{D}$ multirate processing theory, decimators and interpolators. More detail can be found in [1], [5]. We use the following notation: $n=\left[n_{1}, n_{2}\right]^{T}, w=\left[w_{1}, w_{2}\right]^{T}, M=\left[\begin{array}{cc}m_{11} & m_{21} \\ m_{12} & m_{22}\end{array}\right], \sum_{n}=\sum_{n_{1}=-\infty}^{+\infty} \sum_{n_{2}=-\infty}^{+\infty}$.

Downsampling and upsampling of a $2 \mathrm{D}$ signal is possible using different methods and depend on sampling matrix [1], [5]. The most common sampling matrices are the rectangular sampling matrix $M_{r}$ and the quincunx sampling matrices $M_{q}$ :

$$
M_{r}=\left[\begin{array}{ll}
2 & 0 \\
0 & 2
\end{array}\right], \quad M_{q 1}=\left[\begin{array}{rr}
1 & 1 \\
-1 & 1
\end{array}\right], \quad M_{q 2}=\left[\begin{array}{rr}
2 & -1 \\
0 & 1
\end{array}\right]
$$

These sampling matrices define the rectangular and quincunx sampling grids as shown in Fig. 1:
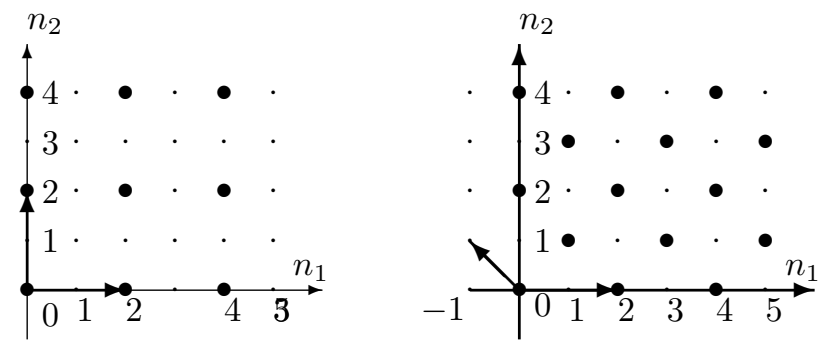

Figure 1: Rectangular and Quincunx Grid Types

Decimation and interpolation are the basic operations in a multirate systems. In a $2 \mathrm{D}$ decimator, shown in Fig. 2(a), the input signal $x(n)$ is lowpass filtered by 
$H(w)$, modulated by $\mathrm{d}(\mathrm{n})$ and then downsampled according to a sampling matrix M. The modulation function can be expressed by [1]

$$
d(n)=\frac{1}{|\operatorname{det} M|} \sum_{l=0}^{|\operatorname{det} M|-1} \exp \left(j 2 \pi k_{l}^{T} M^{-1} n\right)
$$

where $k_{l}$ are the integer vectors inside the fundamental parallelepiped $F P\left(M^{T}\right)$, and $\mathrm{M}$ is the sampling matrix [1], [5]. The output $\mathrm{Y}(\mathrm{w})$ in frequency domain is given by

$$
Y(w)=\frac{1}{|\operatorname{det} M|} \sum_{l=0}^{|\operatorname{det} M|-1} H\left(M^{-T}\left(w-2 \pi k_{l}\right)\right) X\left(M^{-T}\left(w-2 \pi k_{l}\right)\right) .
$$

If the lowpass filter $H(w)$ is ideal with support in a special region, equation $(2.3)$ becomes

$$
Y(w)=\frac{1}{|\operatorname{det} M|} X\left(M^{-T} w\right)
$$

The term $|\operatorname{det} M|$ is the sampling rate reduction factor. Frequency aliasing can be avoided by bandlimiting properly with an anti-alias filter $H(w)$ or making the input signal $X(w)$ with a support inside the symmetric parallelepiped $S P\left(\pi M^{-T}\right)$, defined as [5],

$$
\begin{aligned}
& w=\pi M^{-T} x, \quad x \in[-1,1)^{2} .
\end{aligned}
$$

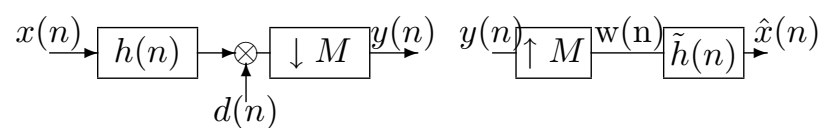

(a)

Figure 2: 2D Decimator and Interpolator

In the 2D interpolator shown in Fig. 2(b), the input signal is upsampled and the resulting signal is filtered by a lowpass filter $\tilde{H}(w)$ in order to filter out the images components and to get the reconstructed signal $\hat{x}(n)$, and the output signal [1] is

$$
\hat{X}(w)=\tilde{H}(w) Y\left(M^{T} w\right)=\tilde{H}(w) \frac{1}{|\operatorname{det} M|} X(w)
$$

and the reconstruction filter should have a gain $|\operatorname{det} M|$, so that $\hat{X}(w) \cong X(w)$.

\section{Two-Dimensional Complementary Filter Banks and Wavelet Theory}

In this section, complementary filters definitions and design are first given, then CF Banks and its relation with wavelet representation are shown. The $2 \mathrm{D}$ complemen- 
tary filters are filters with impulse response defined by

$$
h_{1}\left(n_{1}, n_{2}\right)=\delta\left(n_{1}, n_{2}\right)-h_{0}\left(n_{1}, n_{2}\right),
$$

where $h_{0}\left(n_{1}, n_{2}\right)$ is a lowpass filter and $h_{1}\left(n_{1}, n_{2}\right)$ is its complementary highpass filter.

The designs of rectangular, quincunx and circular complementary filters are done with finite impulse response (FIR), causal, zero-phase filters, i.e., their impulse responses should satisfy the following symmetric property [6]

$$
h\left(n_{1}, n_{2}\right)=h\left(N_{1}-n_{1}, N_{2}-n_{2}\right),
$$

where $0 \leq n_{1} \leq M_{1}, 0 \leq n_{2} \leq M_{2}$, and the length of the FIR filter is $N_{1}=2 M_{1}+1$, $N_{2}=2 M_{2}+1$.

The impulse response of a two-dimensional rectangular lowpass filter is [6]

$$
h_{r}\left(n_{1}, n_{2}\right)=h_{1}\left(n_{1}\right) h_{2}\left(n_{2}\right)=\frac{\sin \left(w_{c_{1}} n_{1}\right)}{\pi n_{1}} \cdot \frac{\sin \left(w_{c_{2}} n_{2}\right)}{\pi n_{2}} .
$$

The highpass rectangular complementary filter impulse response is

$$
h_{r h}\left(n_{1}, n_{2}\right)=\delta\left(n_{1}, n_{2}\right)-\frac{\sin \left(w_{c_{1}} n_{1}\right)}{\pi n_{1}} \cdot \frac{\sin \left(w_{c_{2}} n_{2}\right)}{\pi n_{2}} .
$$

The lowpass quincunx filter is obtained from a linear transformation of the separable lowpass rectangular filter with cutoff frequencies $w_{c_{1}}=w_{c_{2}}=\pi / 2$. The impulse response of the quincunx lowpass filter is obtained from the separable lowpass rectangular filter by

$$
h_{q}\left(n_{1}, n_{2}\right)=h_{r}\left(M_{q} n\right)=2 \frac{\sin \left(\frac{\pi}{2} \cdot\left(n_{1}+n_{2}\right)\right)}{\pi\left(n_{1}+n_{2}\right)} \cdot \frac{\sin \left(\frac{\pi}{2}\left(-n_{1}+n_{2}\right)\right)}{\pi\left(-n_{1}+n_{2}\right)},
$$

where the impulse response coefficients of the lowpass quincunx filters in equation (3.5) were multiplied by $\left|\operatorname{det} M_{q}\right|=2$ to keep the frequency response amplitude correct. The highpass quincunx filter coefficients are found from the definition of complementary filters,

$$
h_{q h}\left(n_{1}, n_{2}\right)=\delta\left(n_{1}, n_{2}\right)-h_{q}\left(n_{1}, n_{2}\right) .
$$

The impulse response of a two-dimensional lowpass circular filter is given by [6]

$$
h_{c}\left(n_{1}, n_{2}\right)=\frac{w_{c}}{2 \pi \sqrt{n_{1}^{2}+n_{2}^{2}}} \mathcal{J}_{1}\left(w_{c} \sqrt{n_{1}^{2}+n_{2}^{2}}\right),
$$

where $\mathcal{J}_{1}(x)$ is the Bessel function of the first kind and first order. The complementary highpass circular impulse response is

$$
h_{c h}\left(n_{1}, n_{2}\right)=\delta\left(n_{1}, n_{2}\right)-\frac{w_{c}}{2 \pi \sqrt{n_{1}^{2}+n_{2}^{2}}} \mathcal{J}_{1}\left(w_{c} \sqrt{n_{1}^{2}+n_{2}^{2}}\right) .
$$

As an example, 31x31 FIR lowpass and highpass complementary rectangular, quincunx and circular filters were designed, and their impulse responses, with $w_{c}=$ $\pi / 2$, are shown in Figs. 3 and 4 . 
Figure 3: Lowpass Rectangular, Quincunx and Circular Filters Impulse Responses

Figure 4: Nonseparable Highpass Complementary Rectangular, Quincunx and Circular Filters Impulse Responses

In Fig. 5, the 2-channel complementary filter bank tree structure is shown. In the analysis, the input signal is filtered with lowpass and highpass filters and then downsampled, using the sampling matrix $\mathrm{M}$, yielding the approximation signal $x l_{r_{l}}$ and the detail signal $x h_{r_{l}}$, respectively. In the synthesis, the signals $x l_{r_{l}}$ and $x h_{r_{l}}$ are upsampled using the sampling matrix $\mathrm{M}$, filtered with lowpass and highpass filters, respectively, then added and multiplied by $|\operatorname{det} M|$ in order to get the reconstructed signal $\hat{x}_{r_{l+1}}$.

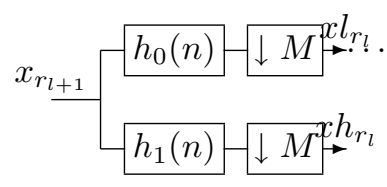

(a) Analysis

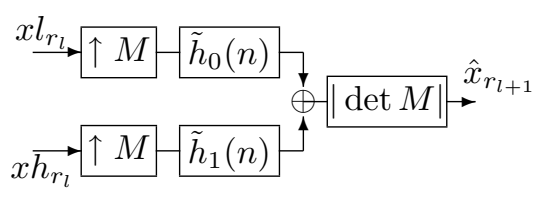

(b) Synthesis

Figure 5: Tree-Structure of 2-Channel CF Bank

For the 2-channel filter banks, perfect reconstruction is obtained when sampling is quincunx and the filter is also quincunx. The synthesis filters should be

$$
\tilde{H}_{0}(w)=2 H_{0}(w),
$$




$$
\tilde{H}_{1}(w)=2 H_{1}(w)
$$

where $H_{0}(w)=H(w)$ is a quincunx lowpass filter and $H_{1}(w)=H(w+K), \mathrm{k}=[\pi, \pi]$ is a highpass filter. Others structures can be developed to obtain perfect reconstruction with rectangular sampling or rectangular and circular filters, at the cost of lower compression [1].

To connect the CF banks to $2 \mathrm{D}$ wavelet representation $[2,3]$, it is considered the discrete approximation of a signal $f(x, y) \in L^{2}\left(R^{2}\right)$ at a series of increasing resolution levels $r_{l}=2^{-l}, l \geq 0$. The approximation function at resolution $r_{l+1}$ is

$$
A_{r l} f=f * \tilde{\phi}_{r_{l}}\left({ }_{r l} n, r_{l} m\right)
$$

where "*" is the $2 \mathrm{D}$ convolution symbol, $\tilde{\phi}_{r_{l}}(x, y)$, the two-dimensional scaling function, corresponds to a 2D lowpass filter.

The difference of information of a signal $f(x, y)$ at resolution $r_{l}=2^{l}$ is

$$
D_{r_{l}} f=f * \tilde{\psi}_{r_{l}}\left(r_{l} n, r_{l} m\right)
$$

where $\tilde{\psi}_{r_{l}}(x, y)$, the two-dimensional wavelet function, corresponds to a 2D highpass filter. Dilating and translating $\phi_{r_{l}}(x, y)$ and $\psi_{r_{l}}(x, y)$, the families of functions with $r_{l}$ build an orthonormal basis on a vector space $V_{r_{l}}, S_{r_{l}}$ such that

$$
V_{r_{l+1}}=S_{r_{l}} \oplus V_{r_{l}}
$$

The set of $2 \mathrm{D}$ orthogonal wavelets decomposition is represented by the two components

$$
\left(A_{r_{L}} f, D_{r_{l}} f\right)_{-L \leq l \leq-1}
$$

These functions, with complementary filter banks, are nonseparable and cover all $[-\pi, \pi]^{2}$ plane, in the analysis.

Using Noble's equivalence [5], the equivalente lowpass filter at the ith-level of the $\mathrm{CF}$ bank is given by [1]

$$
H_{l p}^{(i)}(w)=\prod_{j=0}^{i-1} H\left(\left(M^{T}\right)^{j} w\right)
$$

$i=1,2, \cdots$. A similar function to (3.15) was defined in [4].

The bandpass filter at the ith step is the difference between the complementary highpass filter at that level and the sum of the rest of the bandpass highpass filters of the previous levels, i.e.,

$$
H_{b p}^{(i)}(w)=\left[\left(1-H_{e q}^{(i)}(w)\right)-\left(H_{b p}^{(i-1)}(w)+H_{b p}^{(i-2)}(w)+\ldots+H_{1}(w)\right)\right]
$$




\section{Two-Dimensional Scaling and Wavelet Functions}

The lowpass impulse response in the ith step of the decomposition is the inverse Fourier transform of the equivalent lowpass filter given in equation (3.15),

$$
h_{e q}^{(i)}\left(n_{1}, n_{2}\right)=\mathcal{F}^{-1}\left(H_{e q}^{(i)}(w)\right)=\mathcal{F}^{-1}\left(\prod_{j=0}^{i-1} H\left(\left(M^{T}\right)^{j} w\right)\right),
$$

where $H(w)$ is the original lowpass filter. Then, the $2 \mathrm{D}$ scaling function $\phi^{(i)}\left(n_{1}, n_{2}\right)$ in the ith step is

$$
\phi^{(i)}\left(n_{1}, n_{2}\right)=h_{e q}^{(i)}\left(n_{1}, n_{2}\right) .
$$

The inverse Fourier transform of equation (3.16) is

$$
h_{b p}^{(i)}\left(n_{1}, n_{2}\right)=\delta\left(n_{1}, n_{2}\right)-h_{e q}^{(i)}\left(n_{1}, n_{2}\right)-h_{b p}^{(i-1)}\left(n_{1}, n_{2}\right)-\ldots-h_{1}\left(n_{1}, n_{2}\right) .
$$

Then, the bandpass wavelet at the ith iteration is

$$
\psi_{b p}^{(i)}\left(n_{1}, n_{2}\right)=h_{b p}^{(i)}\left(n_{1}, n_{2}\right) .
$$

If it is considered 2-level CF bank decomposition using quincunx sampling and filters, the scaling and wavelet functions are

$$
\begin{aligned}
& \phi^{(2)}\left(n_{1}, n_{2}\right)=h_{e q}^{(2)}\left(n_{1}, n_{2}\right)=\frac{\sin \left(\frac{\pi}{2} n_{1}\right)}{\pi n_{1}} \frac{\sin \left(\frac{\pi}{2} n_{2}\right)}{\pi n_{2}}, \\
& \psi_{01}\left(n_{1}, n_{2}\right)=h_{b p}^{(2)}\left(n_{1}, n_{2}\right)=-\frac{\sin \left(\frac{\pi}{2} n_{1}\right)}{\pi n_{1}} \frac{\sin \left(\frac{\pi}{2} n_{2}\right)}{\pi n_{2}} \\
& +2 \frac{\sin \left(\frac{\pi}{2}\left(n_{1}+n_{2}\right)\right)}{\pi\left(n_{1}+n_{2}\right)} \frac{\sin \left(\frac{\pi}{2}\left(-n_{1}+n_{2}\right)\right)}{\pi\left(-n_{1}+n_{2}\right)}, \\
& \psi_{1}\left(n_{1}, n_{2}\right)=h_{1}^{(1)}\left(n_{1}, n_{2}\right)=\delta\left(n_{1}+n_{2}\right) \\
& -2 \frac{\sin \left(\frac{\pi}{2}\left(n_{1}+n_{2}\right)\right)}{\pi\left(n_{1}+n_{2}\right)} \frac{\sin \left(\frac{\pi}{2}\left(-n_{1}+n_{2}\right)\right)}{\pi\left(-n_{1}+n_{2}\right)} .
\end{aligned}
$$

In Fig. 6 and 7, 31x31 scaling and bandpass wavelet functions and their magnitude are shown, respectively, for 2-level CF Banks decomposition using quincunx sampling and filters.

Figure 6: Scaling2, Wavelet2 and Wavelet1 functions, respectively, in 2-level decomposition, Quincunx filter and sampling. 
Figure 7: Scaling2, Wavelet2 and Wavelet1 magnitudes, respectively, in 2-level decomposition, Quincunx filter and sampling.

\section{Conclusion}

In this paper we proposed a $\mathrm{CF}$ bank which can be used to get $2 \mathrm{D}$ scaling and bandpass wavelet functions. The $2 \mathrm{D}$ multirate theory permite us to have a variety of sampling and filtering methods. The CF bank was related to wavelet representation. When CF Banks is implemented using quincunx sampling and filters, perfect reconstruction is achieved, and although for other cases perfect reconstruction is not reached, the analysis is done free of aliasing. It was shown that 2D scaling and bandpass wavelet functions can be found iteratively, through the equivalent transfer functions at the ith-level of CF Banks decomposition. An illustration of these functions was shown for 2-level decomposition case. The CF banks can be applied also for analysis/synthesis of signals, image compression and edge detection.

\section{References}

[1] M.G. Almeida, "Two-Dimensional Complementary Filter Bank in the Wavelet Representation Context: Theory, Design and Applications", Ph.D. Dissertation, University of Pittsburgh, 1994.

[2] S. Mallat, A Theory for Multiresolution Signal Decomposition: The Wavelet Representation, IEEE Transactions on Pattern, Analysis, and Machine Intelligence, 11 No.7 (1989), 674-692.

[3] S. Mallat, Multifrequency Channel Decompositions of Images and Wavelet Models, IEEE Transactions on Acoustic, Speech, and Signal Processing, 37 No.12 (1991), 2091-2110.

[4] J. Kovačević, M. and Vetterli, Nonseparable Multidimensional Perfect Reconstruction Filter Banks and Wavelet Bases for $R^{n}$, IEEE Transactions on Information Theory, 38 No.2 (1992), 533-555.

[5] P.P. Vaidyanathan, "Multirate Systems and Filter Banks", Englewood Cliffs, NJ: Prentice Hall, 1993.

[6] J.S. Lim, "Two-Dimensional Signal Processing and Image Processing", Prentice Hall Signal Processing Series, A. Oppenheim, 1990. 